\title{
Monitoring Ischemic Cerebral Injury in Spontaneously Hypertensive Rats by Diffusion Tensor Imaging
}

\author{
Gregory C. KUJOTH ${ }^{1}$, Gabriel F. NEVES ${ }^{1}$, Ulas CIKLA ${ }^{1}$, Erinc AKTURE1 ${ }^{1}$, Kutluay ULUC ${ }^{1}$, Chihwa SONG², \\ Tomer HANANYA ${ }^{1}$, Alireza SADIGHI ${ }^{1}$, Peter FERRAZZANO ${ }^{3,4}$, Mustafa K. BASKAYA ${ }^{1}$ \\ ${ }^{1}$ University of Wisconsin, School of Medicine and Public Health, Department of Neurological Surgery, Madison, WI, USA \\ ${ }^{2}$ University of Wisconsin, School of Medicine and Public Health, Wisconsin Institutes for Medical Research, Department of Medical Physics, \\ Madison, WI, USA \\ ${ }^{3}$ University of Wisconsin, School of Medicine and Public Health, Department of Pediatrics, Madison, WI, USA \\ ${ }^{4}$ University of Wisconsin, The Waisman Center, Madison, WI, USA
}

\section{ABSTRACT}

AIM: We have applied diffusion tensor imaging (DTI) to interrogate microstructural changes in white matter integrity in a widely used middle cerebral artery occlusion (MCAO) model of cerebral ischemia.

MATERIAL and METHODS: We performed ex vivo DTI 35 days after 60 minutes transient focal ischemia in male spontaneously hypertensive rats and generated fractional anisotropy (FA), mean, axial and radial diffusivity maps. Regions of interest corresponding to external capsule (EC), corpus callosum (CC) and internal capsule (IC) were compared among sham and stroked rats. We compared tractographic projections of white matter fiber patterns and examined white matter integrity by Luxol fast blue histological analysis. We also determined infarct lesion volumes at 24 hours post-ischemia by T2-weighted magnetic resonance imaging (MRI) or at 35 days by histological staining with cresyl violet.

RESULTS: We found alterations in EC and IC, but not CC, as represented by decreased FA and increased mean, axial and radial diffusivities. The size of the ischemic lesion detected subacutely by T2-weighted MRI or at 35 days by histological staining correlated with the decline in FA in the affected structures. Tractography revealed disruption of fiber trajectories through the EC and reorientation of fibers within the caudate/putamen of rats subjected to MCAO. Similarly, loss of white matter integrity in the EC and increased white matter density in the caudate/putamen along the infarct border zone was evidenced by Luxol fast blue staining.

CONCLUSION: Diffusion tensor imaging therefore allows for monitoring of white matter injury and reorganization in hypertensive rats.

KEYWORDS: Diffusion tensor imaging, Middle cerebral artery occlusion, Cerebral ischemia, External capsule, Internal capsule

Abbreviations: AD: Axial diffusivity, CC: Corpus callosum, CCA: Common carotid artery, DTI: Diffusion tensor imaging, EC: External capsule, ECA: External carotid artery, FA: Fractional anisotropy, IC: Internal capsule, ICA: Internal carotid artery, MCAO: Middle cerebral artery occlusion, MD: Mean diffusivity, PBS: Phosphate-buffered saline, RD: Radial diffusivity, SHR: Spontaneously hypertensive rats. 


\section{INTRODUCTION}

Non-invasive imaging of the central nervous system (CNS) has advanced significantly over the last several decades with the development of a variety of magnetic resonance-based imaging (MRI) modalities. Conventional protocols include T1-weighted imaging, which provides anatomical detail with good resolution, and T2-weighted imaging, which can detect the location of edematous tissue from ischemic damage or traumatic brain injury, as well as reveal the presence of brain tumors. Diffusion-weighted imaging is highly sensitive to the early hyper-acute effects of cerebral ischemia and has been combined with perfusion-weighting to estimate the boundaries between ischemic core and penumbra (potentially salvageable tissue), although this approach has some pitfalls $(12,50)$.

Diffusion tensor imaging (DTI) is an extension of diffusion weighting that is used to examine the structure and integrity of white matter tracts (36). Diffusion-weighted imaging adds a complementary pair of pulsed magnetic gradients to the MRI sequence prior to signal acquisition. The first pulse generates spatial encoding and the second pulse removes it, such that the net signal generated by stationary molecules is unaffected. If molecules move during the interval between the two gradient pulses, however, the spatial encoding cannot be completely removed and some signal is lost-the greater the diffusion, the greater the signal attenuation. DTI collects a series of diffusion-weighted images using paired pulsed gradients applied in multiple non-colinear orientations and uses these data to construct a mathematic model of the three-dimensional diffusion of water, the diffusion tensor (2). The diffusion tensor ranges from spherical to highly ellipsoid, depending upon how highly directional is the water diffusion in the tissues. One convenient measure derived from the diffusion tensor is fractional anisotropy (4), which is an indicator of the overall extent of directional water movement, with values ranging between $0-1$ (higher represents greater anisotropy). Fractional anisotropy is frequently employed because it is rotationally invariant. The tissue architecture of the central nervous system (e.g. cell membranes and myelin fibers) produces greater barriers to diffusion occurring perpendicular to the orientation of the nerve fibers, relative to diffusion along the lengths of the fibers (37). By taking advantage of this characteristic, DTI can be used to determine the directionality of white matter structures within a given voxel and map nerve fiber tracts within the brain and spinal cord $(3,36)$. This allows for an assessment of the extent of damage to such structures following ischemia or traumatic brain injury. Key advantages of DTI over histological methods of white matter assessment include the ability to image an intact brain, avoiding the potential distortions of tissue architecture that may result from sectioning, and the ability to produce tractographic projections of nerve fiber orientation and organization.

DTI is being increasingly applied to animal models of CNS injury, including those involving neonatal hypoxia-ischemia and stroke $(8-10,15,43,62)$. Previous rodent studies have examined white matter injury following experimental focal ischemia in normotensive rats $(25,43,44,62)$ or studied spontaneous changes in DTI in hypertensive rats $(6,35)$. As far as we are aware, no reports have applied DTI to hypertensive rats that have been subjected to MCAO. Hypertension is a major risk factor for human stroke $(19,40)$ and hypertensive rats can have an increased susceptibility to focal ischemic injury relative to normotensive strains $(13,16,17)$. In this report, we have utilized DTI to examine white matter injury following transient focal ischemia in spontaneously hypertensive rats (SHRs). We find clear evidence of injury to the EC and IC 35 days after stroke and correlate this to other assessments of cerebral injury, such as T2-weighted MRI (T2w-MRI) and histological staining. Finally, we apply tractography to visualize three-dimensional patterns of white matter tract organization after transient ischemic injury.

\section{MATERIAL and METHODS}

\section{Animals and Middle Cerebral Artery Occlusion}

All animal experiments were performed under protocols approved by Animal Care and Use Committees of the University of Wisconsin-Madison. Spontaneously hypertensive rats (SHR/NCrl) were obtained from Charles Rivers Laboratories (Wilmington, MA, USA). MCAO surgery was performed similarly to that described previously $(33,60)$. Rats were anesthetized with isoflurane and the presumptive incision area was shaved and disinfected with Betadine, prior to administration of $0.2 \mathrm{ml} 0.5 \%$ bupivacaine for local analgesia. Under an operating microscope, the left common carotid artery (CCA), external carotid artery (ECA) and internal carotid artery (ICA) were exposed through a ventral midline incision, dissected free from the surrounding fascia and nerves and the ECA was tied off with a silk thread. Micro-aneurysm clips were temporarily placed on the CCA and ICA and a small arteriotomy was made on the ECA. An approximately $20 \mathrm{~mm}$ length of silicon-coated monofilament nylon suture (Doccol Corporation, Sharon, MA) was inserted through the arteriotomy on the ECA and advanced into the ICA to the origin of the MCA, effectively occluding the MCA for 1 hour. The remaining microclip was removed from the CCA and the surgical incision was temporarily closed using a silk thread suture. After $1 \mathrm{~h}$ of occlusion, the incision was reopened and the intraluminal nylon suture was withdrawn to allow for reperfusion (the ECA was permanently tied off with silk thread suture). Prior to closing, $1 \mathrm{ml}$ sterile saline was added to the incision region to reduce dehydration and the site was closed with suture. Body and cranial temperatures were maintained by placing the rat on a thermostat-controlled water-filled heating pad at $37-38^{\circ} \mathrm{C}$ during surgery. Sham-operated controls received midline neck incision and dissection without arteriotomy, followed by subsequent closure with silk thread suture. All rats received post-surgical analgesia by intraperitoneal injection of buprenorphine $(0.05 \mathrm{mg} / \mathrm{kg}$ body weight). Total surgery time, including 60 min occlusion period, was less than $2 \mathrm{~h}$.

Data presented in this study come from a larger unpublished project investigating the effects of injecting human embryonic stem cell-derived mesenchymal stromal cells (MSCs; $2 \times 10^{7}$ cells), or MSC-conditioned culture medium (CM) on the in vivo response to MCAO. Rats injected with Dulbecco's phosphate- 
buffered saline (DPBS) or unconditioned culture media (UCM, complete Dulbecco's modified Eagle's medium) were used as control groups. All injections $(1 \mathrm{ml})$ were done in the femoral vein, at $24 \mathrm{hr}$ post-MCAO, shortly after the T2 image acquisition timepoint. In total, 70 rats were subjected to MCAO and received injections. Of these, 68 rats were subjected to T2w-MRI at $24 \mathrm{hr}$, which revealed 10 rats that had failed to develop infarcts with clear cortical involvement and thus were excluded from the study. Fourteen rats died prior to the end of the study (20\% mortality) and were excluded. Infarct volume data presented in the bar graph figure correspond to DPBS group rats for which infarct data were measured at all three time points by either T2w-MRI or cresyl violet staining $(\mathrm{N}=7)$. Ex vivo DTI was performed on a total of 20 rats subjected to MCAO ( 5 from each treatment group), as well as on 4 sham control rats. Ipsilateral and contralateral DTI bar graph data were taken from the DPBS control group only $(\mathrm{N}=5)$, labeled as "MCAO" in the bar graph figure, and the sham rats $(\mathrm{N}=4)$. Correlation analyses used combined data from DPBS-, MSC-, $\mathrm{CM}$-, and UCM-injected rats (total $\mathrm{N}=20$ ).

\section{In vivo T2-Weighted Magnetic Resonance Imaging}

Rat MRI was performed with the assistance of the University of Wisconsin Small Animal Imaging Center. Rats were anesthetized with isoflurane (1-3\%) and positioned in a Varian 4.7T small animal MRI scanner using a Varian rat radiofrequency coil. The animal's respiratory rate was monitored during scanning and body temperature was controlled using either a water circulating heating pad or a warm blown air system.

Rats were imaged at approximately 18-24 $\mathrm{h}$ and 5 days after MCAO. T2-weighted fast spin echo images (TR $=3500 \mathrm{~ms}$; effective TE $=67.54 \mathrm{~ms}$; echo train length $=8$; matrix size $=128 \times 128$; number of averages $=8$ ) were acquired from 17 contiguous axial slices with a field of view of $17 \times 17 \mathrm{~mm}$ and a slice thickness of $1 \mathrm{~mm}$. T2 acquisition time was 287 $\mathrm{s}$ and total time in the magnet was 7 min per animal. Masks of healthy (un-infarcted) tissue in ipsilateral and contralateral hemispheres were created for each slice showing an infarct using Image $J$ and infarcted tissue volumes were calculated using the indirect method, which reduces the varying effects of edema on infarct volumes during sequential time-points $(31,58)$ : healthy contralateral area minus healthy ipsilateral area, with the difference divided by the healthy contralateral area for each slice; the resulting area values were multiplied by the distance between sections and summed across all slices to determine the infarct volume, expressed as a percentage of the contralateral hemisphere (\%CLH).

\section{Tissue Harvesting and Cresyl Violet Staining}

At 5 weeks post-MCAO, rat brain tissues were fixed in situ under deep isoflurane anesthesia by intracardial perfusion with freshly prepared $4 \%$ paraformaldehyde in $1 \mathrm{X}$ phosphatebuffered saline (PBS). Subsequently, the brains were extracted and post-fixed in $4 \%$ paraformaldehyde for at least 3 days prior to placement into $30 \%$ sucrose solution (in 1X PBS) for longer term storage at $4^{\circ} \mathrm{C}$, if needed. At least $24 \mathrm{~h}$ prior to ex vivo DTI (see below), brains were transferred back into 1X PBS to fully hydrate the samples.
After DTI was completed, the rat brains were returned to $30 \%$ sucrose until further processing. Rat brains were cut into 35 $\mu \mathrm{m}$ sections using a freezing microtome and the slices were transferred into freezing solution (30\% ethylene glycol, 30\% glycerol in $1 \mathrm{X}$ PBS) for storage at $-20^{\circ} \mathrm{C}$. Sections used for histological staining were either mounted directly after cutting or thawed, rinsed three times in 1X PBS and mounted.

Cresyl violet (Nissl) staining was used to histologically assess the extent of cerebral infarction at the study endpoint of 5 weeks post-MCAO. Tissue sections went through sequential cycles (2 min each) of defatting in $70 \%, 95 \%$, and $100 \%$ ethanol, followed by three cycles in $95 \%, 70 \%$ ethanol and water, prior to staining with $0.5 \%$ cresyl violet (C50242, Sigma Chemical Company, St. Louis, MO, USA). The sections were differentiated in $70 \%, 95 \%$, and $100 \%$ ethanol (30 sec each), prior to a 2 min wash in $100 \%$ ethanol. Following two rinses in xylene (1 min each), the sections were mounted and coverslipped. Images were acquired using a flatbed scanner and analyzed with Image J using the indirect method to determine infarct volume, as described above for T2-weighted images.

\section{Luxol Fast Blue Staining}

Luxol fast blue staining was performed to visualize white matter structures through the detection of myelin. Frozen brain sections were obtained as described above, rinsed three times in 1X PBS and mounted. Sections were treated overnight in $0.1 \%$ Luxol Fast Blue Stain Solution (Newcomer Supply, Middleton, WI) at $60^{\circ} \mathrm{C}$, followed by $2-3$ rinses each in $70 \%$ ethanol and water. Tissues were individually differentiated in $0.5 \%$ lithium carbonate for approximately 30 seconds, followed by 2 rinses in $70 \%$ ethanol for 1 minute each or until gray and white matter could be distinguished. Sections were rinsed briefly in water, counterstained in $0.5 \%$ cresyl violet for $1 \mathrm{~h}$ at room temperature and then rinsed twice in $100 \%$ ethanol and once in xylene for 1 minute each, prior to coverslip mounting. Images were acquired using a Keyence BZ9000 microscope at 4X and stitched in BZ Analyzer II software to produce a view of the entire section. High power images were acquired at 20X magnification.

\section{Ex-vivo Diffusion Tensor Imaging}

Preliminary trials employing echo planar imaging sequences (required to keep scan times short enough to be practical) for in vivo DTI yielded relatively poor quality, low resolution images using the Varian MRI system available to us; these images were not readily amenable to analysis using defined white matter regions of interest. We therefore pursued exvivo imaging in order to be able to employ considerably longer spin echo pulse sequences to generate images with significantly improved resolution. Paraformaldehyde-fixed rat brains were rehydrated overnight in 1X PBS and positioned inside a syringe filled with fluorinert FC-3283 solution at room temperature within a Varian mouse radiofrequency coil. A pulsed gradient multi-slice spin echo pulse sequence was employed ( $T E=24.17 \mathrm{~ms}$; $T R=2000 \mathrm{~ms}$; matrix size = $256 \times 256$; number of averages $=3$; 30 slices; field of view of $32 \times 32 \mathrm{~mm}$; $0.5 \mathrm{~mm}$ slice thickness; diffusion gradient = $22.80 \mathrm{G} / \mathrm{cm}, \delta=4.50 \mathrm{~ms}, \Delta=10.80 \mathrm{~ms}$; b-value $=1007.90 \mathrm{~s} /$ 
$\mathrm{mm}^{2}$ ). For each axial slice, 3 non-diffusion-weighted images and 30 diffusion-weighted images with distinct and uniformly distributed diffusion gradient directions at the same $b$-value of $1007.90 \mathrm{~s} / \mathrm{mm}^{2}$ were acquired, as suggested by Jones et al. (24). Fractional anisotropy, mean diffusivity, axial diffusivity and radial diffusivity maps were created using FMRIB's Diffusion Toolbox, (FSL, Oxford, UK). Images were eddy current corrected to remove gradient coil distortions, and a binary brain mask generated. Computation of the diffusion tensor was performed using HI-SPEED software (http://sites.google. com/site/hispeedpackets/), which utilizes the constrained nonlinear least squares method of estimation as described in Koay et al. (28) to ensure that the estimated eigenvalues of the diffusion tensor are non-negative.

For DTI analysis, region of interest masks of ipsilateral and contralateral CC, EC and IC were manually drawn on each slice of the non-diffusion-weighted image using ImageJ (http://rsb.info.nih.gov/ij/) software and the masks then applied to the FA and diffusivity maps. Standardized criteria based on the healthy contralateral image were established to determine which optical slices to evaluate for each white matter structure, such that DTI sets being compared were similar for a given structure. Specifically, regions of interest were created on standardized sets of slices according to the following criteria: CC \& EC, beginning with the first slice to show a contiguous EC-CC structure and ending with the last slice prior to separation of the corpus callosum (10-14 slices per brain); IC, beginning with the first slice with an apparent IC in the healthy contralateral hemisphere and ending with the last slice prior to apparent merging of the IC and cerebral peduncle (6-8 slices per brain). Because loss of white matter integrity was visually obvious, but would not necessarily be reflected if only the remaining healthy white matter were used to calculate mean values, we based ipsilateral structural regions of interest on their mirrored healthy contralateral counterparts, subjected to refinement to better match the contours of the existing ipsilateral structures. All region of interest masks were reviewed by at least two individuals. Mean values were calculated based on the sum of all slices evaluated for a given structure. Relative FA is derived from the ratio of ipsilateral mean FA to contralateral mean FA. There were no significant differences in the contralateral hemisphere FA values between sham controls and the MCAO group so we used relative FA to correlate white matter injury to the infarct size determined by T2-weighted imaging or cresyl violet staining in Figure 4.

Fiber tractography of sham and MCAO rat brains was obtained using the deterministic fiber tracking algorithm in DSI Studio (68). Fiber tracking was seeded with the corpus callosum regions of interest used in the measurement of diffusivity parameters and propagated until no orientation was found in the propagation direction. A maximum turning angle of $60^{\circ}$ was used with a step size of $0.62 \mathrm{~mm}$ (i.e., half of the spatial resolution).

\section{Statistical Analysis}

Statistical analyses were performed in Prism 6 (GraphPad, La Jolla, CA). Infarct volumes were compared using repeated measures one-way analysis of variance, followed by Tukey's post-tests. Diffusivity and anisotropy parameters were compared by repeated measures two-way analysis of variance, followed by Šídák's post-test with correction for multiple comparisons using a family-wide alpha $=.05$. Mean \pm standard deviation values are presented. Correlation analyses were calculated using Pearson's correlation coefficient and best fit linear regressions with the 95\% confidence interval of the best fit are shown in the plots. $P$ values for the correlation coefficients are uncorrected for multiple comparisons. The significance threshold to maintain a family-wise alpha of 0.05 across 12 comparisons (which includes the nine correlations shown, as well as the correlation calculations among infarct volumes and corpus callosum relative FA that are not shown) would be $\mathrm{P}=.00427$ using Šídák's correction (1).

\section{RESULTS}

\section{Determination of Infarct Volumes}

Spontaneously hypertensive rats subjected to $60 \mathrm{~min}$ of MCAO (using the intraluminal suture model $(29,34,60)$ ) were assessed for the extent of infarct development approximately 18-24 $\mathrm{h}$ after surgery using T2w-MRI; a subsequent T2w-MRI was also performed 5 days after surgery. At $24 \mathrm{~h}$ post-MCAO, a sharply demarcated region of increased T2 signal intensity was clearly seen in the ipsilateral hemisphere (Figure 1A). While the infarct lesion generally appeared brighter on images acquired at 5 days post-MCAO (Figure 1B), there was no difference in mean infarct volumes between $24 \mathrm{~h}$ and 5 days post-surgery (Figure $2 \mathrm{~A} ; \mathrm{P}=.8730$ ). Final infarct volumes were determined histologically 35 days after surgery by staining with cresyl violet (Nissl stain) (Figure 1C). Mean infarct volumes assessed histologically were not significantly different from T2-weighted MRI-based estimations at the two earlier time points (Figure 2A; $\mathrm{P}=.3248$, T2w $24 \mathrm{hr}$ vs $\mathrm{CV}$; $\mathrm{P}=.2053$, T2w $120 \mathrm{hr}$ vs CV). We also observed good correlation between T2w-MRI-derived and CV-derived infarct volume estimates among individual rats for all three time point comparisons (Figure 2B).

\section{Ex-vivo Diffusion Tensor Imaging}

In order to determine the extent to which $60 \mathrm{~min}$ of MCAO resulted in loss of white matter integrity, we performed exvivo diffusion tensor $\mathrm{MRI}$ on fixed brains harvested from rats 35 days after sham or MCAO surgery. We examined white matter structures, such as the corpus callosum (CC), external capsule (EC) and internal capsule (IC), for changes in multiple DTI parameters including fractional anisotropy (FA), mean diffusivity (MD), axial diffusivity (AD; also called $\left.\lambda_{\|}\right)$and radial diffusivity (RD, also called $\left.\lambda_{\perp}\right)$ (Figure $\left.3 A-E\right)$.

We observed a decrease in FA values in the ipsilateral EC and IC of rats subjected to $\mathrm{MCAO}$, indicating less directional water diffusion (Figure 4A; MCAO ipsi vs contra, EC $\mathrm{P}=.0012$, IC $\mathrm{P}=.0119$; sham ipsi vs $\mathrm{MCAO}$ ipsi, EC $\mathrm{P}<.0001$, IC $\mathrm{P}=.0191$ ). Additionally, these regions demonstrated an increase in mean diffusivity (Figure 4B; MCAO ipsi vs contra, EC P=.0098, IC $\mathrm{P}=.0015$; sham ipsi vs MCAO ipsi, EC $\mathrm{P}=.0017$, IC $\mathrm{P}<.0001$ ). This pattern of increased mean diffusion with decreased directional diffusion is commonly seen in sub-acute to early 

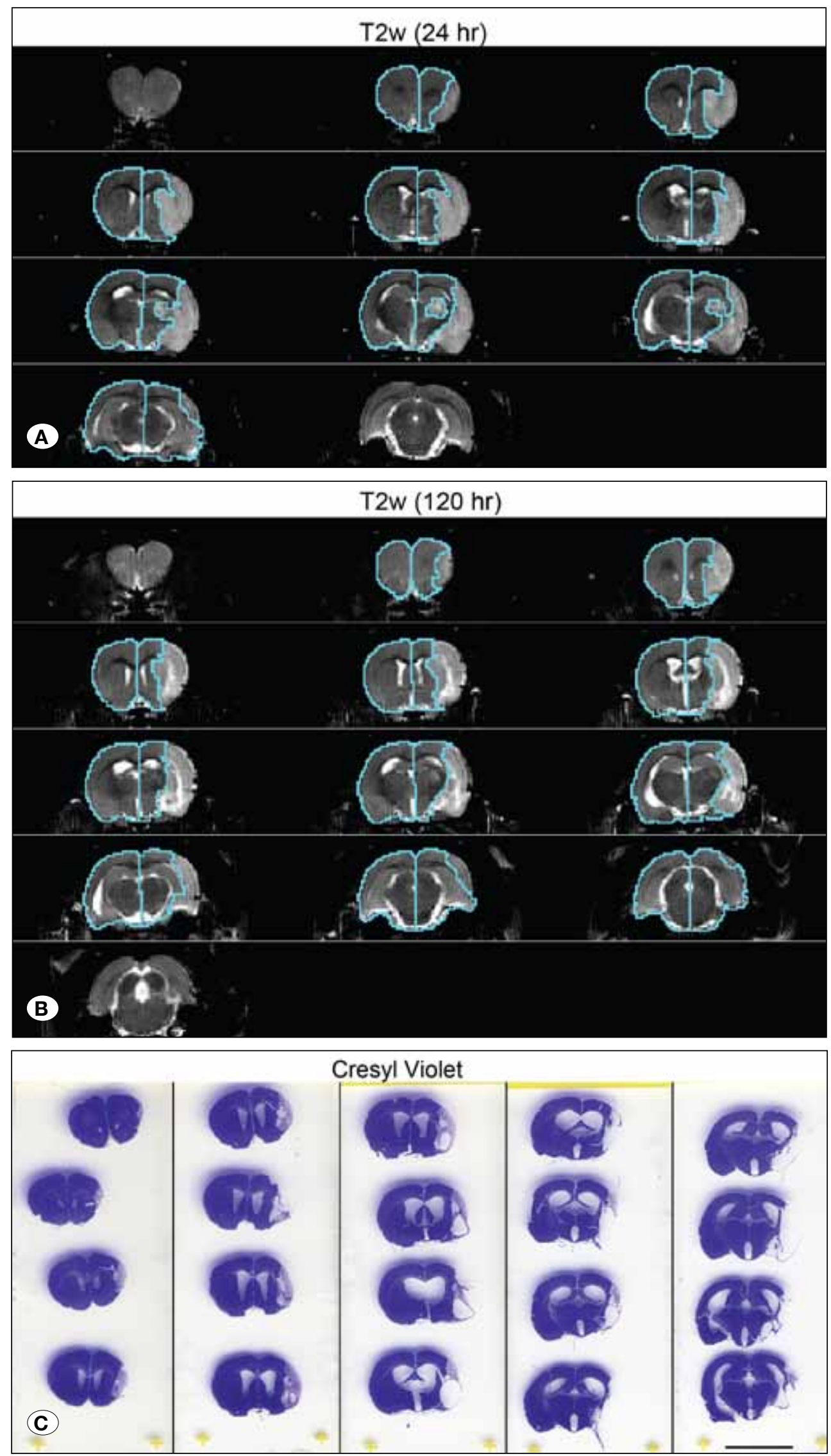

Figure 1: Regions of interest used to calculate infarct volumes in representative T2-weighted and cresyl violet-stained axial brain images from the same individual rat subjected to 60 min left MCAO. (A) T2-weighted images at $24 \mathrm{~h}$ post-MCAO.

(B) T2-weighted images at 5 days (120 h) post-MCAO.

(C) Cresyl violet-stained images at 35 days post-MCAO. Regions of healthy hemispheric area used to calculate infarct volume are shown. 


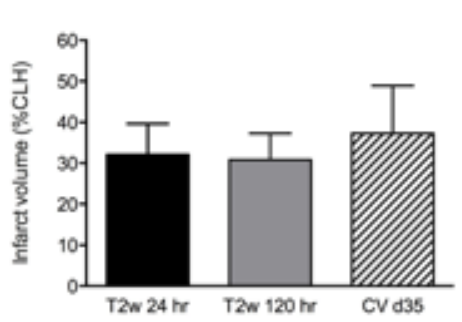

(A)
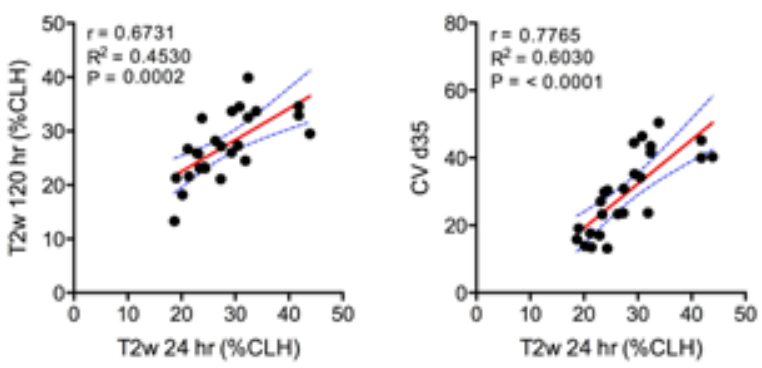

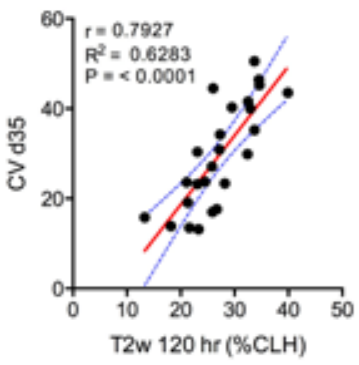

Figure 2: (A) Mean infarct volumes assessed by T2w-MRI at $24 \mathrm{~h}$ or 5 days ( $120 \mathrm{~h}$ ), and by cresyl violet staining at 35 days after MCAO (CV d35). Infarct volumes were calculated using the indirect method and are presented as a percentage of the healthy contralateral hemisphere. Error bars represent standard deviations. (B) Correlations among infarct volumes determined by T2w-MRI at $24 \mathrm{~h}$ or 120 $\mathrm{h}$ after MCAO and by cresyl violet staining at 35 days after surgery. Pearson's correlation coefficients were calculated for the indicated pairs of measurements. The best fit linear regressions (red) and their 95\% confidence intervals (blue dotted lines) are also shown. \%CLH, percent contralateral hemisphere volume.

chronic stages after ischemic injury $(7,15,32,53)$. In order to determine whether the decrease in FA we observed in the ipsilateral IC and EC was due to demyelination or loss of axonal fibers, we next determined the axial and radial diffusivity values in each region. Radial diffusivity increased in the ipsilateral EC and IC in rats subjected to MCAO (Figure 4C; MCAO ipsi vs contra, EC $\mathrm{P}=.0070$, IC $\mathrm{P}=.0008$; sham ipsi vs MCAO ipsi, EC $\mathrm{P}=.0010$, IC $\mathrm{P}<.0001$ ), suggesting that demyelination contributed to the loss of anisotropy that we observed. Interestingly, we also observed an increase in axial diffusivity in the IC and EC (Figure 4D; MCAO ipsi vs contra, EC $\mathrm{P}=.0205$, IC $\mathrm{P}=.0423$; sham ipsi vs MCAO ipsi, $E C P=.0046$, IC $P=.0004$ ), suggesting a component of axonal degeneration. We found no differences in FA, MD, AD or RD among contralateral versus ipsilateral corpus callosum in stroked or sham rats.

The extent of white matter injury as determined by decreased FA at day 35 was correlated to the lesion size determined by T2w-MRI at 5 days post-surgery for both EC and IC (Figure 5) Although below $P=.05$, the correlation coefficient for the comparison between EC FA and $24 \mathrm{~h} \mathrm{T2w-MRI} \mathrm{infarct} \mathrm{lesions}$ did not reach statistical significance when corrected for multiple comparisons (Figure 5A). Loss of FA also correlated with histological assessment of infarct size by cresyl violet staining for EC (Figure 5A). No correlation between relative FA and lesion size as determined using either T2w-MRI or CV was observed for CC (data not shown).

\section{Fiber Tractography}

We next employed tractographic projection using a deterministic algorithm to visualize white matter topology in stroked versus sham rat brains. We seeded the projections using the $\mathrm{CC}$ regions of interest that we outlined for the two-dimensional DTI analyses. Loss of fiber tracts through the infarcted regions was readily apparent in rats subjected to MCAO (Figure 6). We also observed a reorientation of fibers in the ipsilateral caudate/putamen of stroked rats compared to the pattern present in sham animals, suggestive of white matter reorganization along the boundary regions of the infarct.

\section{Histological Assessment of White Matter Integrity}

Finally, we corroborated our DTI results by examining fixed brain slices for white matter integrity using Luxol Fast Blue, which stains myelin basic protein in the nerve sheath. We saw well-defined white matter structures, including EC, CC, IC, anterior commissure, lateral olfactory tract and the optic tract in brain sections from sham rats. Brain sections from rats subjected to MCAO, however, displayed severe disruptions in the EC, and in some cases, posterior portions of the anterior commissure, 35 days after surgery (Figure 7A-E). Additionally, we also observed extensive myelin staining in the caudate/ putamen along the infarct boundary zone in the stroked rats. These patterns were consistent with those observed by fractional anisotropy and tractography analyses of the DTI data.

\section{DISCUSSION}

The use of DTI to predict functional outcome has been applied in the clinical setting. Reduction in relative FA, particularly in the corticospinal tract, within the first month after stroke has been associated with poor motor recovery in human patients at 3 months $(21,38,59)$ or at 2 years $(45)$. Furthermore, poststroke therapeutic interventional studies have shown an association between FA changes and functional improvement. For example, transcranial direct current stimulation combined with physical and occupational therapy can improve upper extremity motor function and this has been correlated with an increase in ipsilateral FA of the alternate motor fibers of these patients (71). Such increased FA might arise from an increased number of aligned fibers or greater myelination and increased FA with therapy seems to be a common finding (18, 49). Similarly, tractography (instead of FA) has been used to assess involvement of specific fiber regions in stroke patients and here too, damage to the IC or other portions of the corticospinal tract is predictive of poor motor outcome at 3 months (46). Likewise, studies in animal models have shown correspondence between DTI measures and behavioral outcomes $(8,10,62)$. 

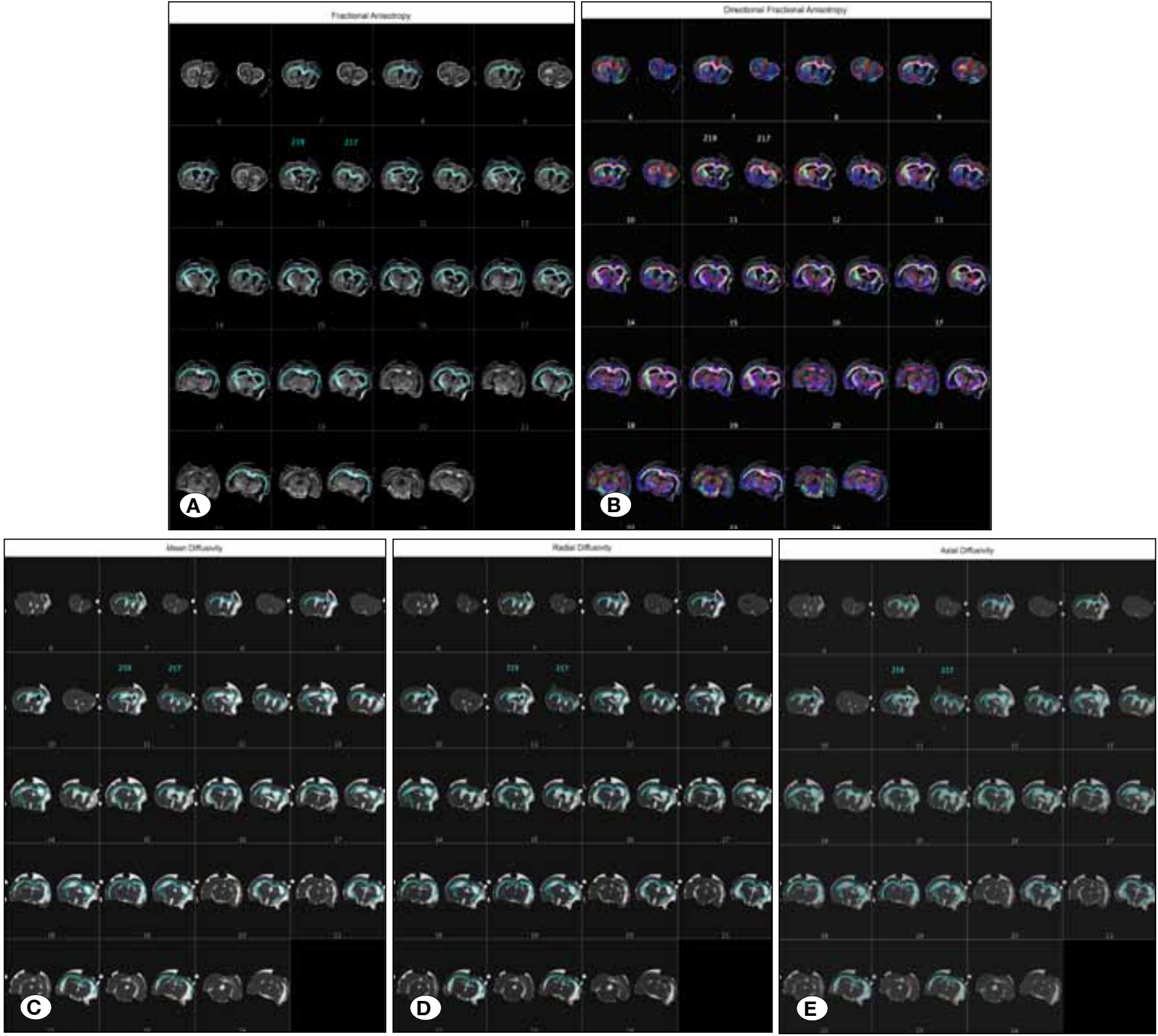

Figure 3: Regions of interest used to calculate fractional anisotropy (A), directionality-encoded fractional anisotropy (B), mean diffusivity (C), radial diffusivity (D), and axial diffusivity (E) in representative axial images from the same rats obtained 35 days after MCAO. Two rats were scanned simultaneously during ex vivo DTI; the rat brain on the right (217) corresponds to the rat brain imaged in Figure 1. Regions of interest corresponding to corpus callosum, EC and IC are outlined in blue or white and used for measuring changes in DTI parameters. Note that cysts and post-mortem tissue cracks identified on the mean diffusivity image were excluded from the regions of interest to avoid artificial skewing of the diffusion measurements. Colors in the directionality-encoded FA image represent lateral-medial (red), dorsal-ventral (green), and rostral-caudal (blue) axes.

In this study, we have employed ex-vivo DTI to examine microstructural changes in white matter following transient focal ischemia in SHRs. Key advantages of DTI over histological methods of white matter assessment include the ability to image an intact brain, avoiding the potential distortions of tissue architecture that may result from sectioning, and the ability to produce tractographic projections of nerve fiber orientation and organization. While the use of ex vivo DTI instead of in vivo DTI surrenders the ability to do longitudinal imaging in individual animals, in our hands, it has the advantages of better resolution, higher signal-to-noise ratio and fewer motion artifacts (data not shown and 41). Nevertheless, we don't claim that in vivo DTI could not be suitable in other cases, depending upon the specific hardware systems available. Although there can be differences in absolute diffusivity values obtained between in vivo and ex vivo DTI $(26,47,55)$, a number of studies have reported that fractional anisotropy may be relatively unaffected by tissue fixation $(14,20,54,56,57$, 
70); fixation has also been reported to decrease FA, however (47). Even if fixation may alter absolute diffusivity parameters, ex vivo DTI can still provide useful indications of white matter injury that are supported by histological evaluation $(8,27,30$, 48). The good agreement between the pattern of FA reduction that we observe in our ex vivo study with that in human stroke patients assessed by in vivo DTI $(64,66)$ supports the general applicability of the ex vivo approach.
In contrast to several previous studies, which measured diffusion changes using small spot regions of interest loosely positioned within a given target structure or using larger geometric shapes spanning multiple structures $(22,43,61$, $63)$, we have analyzed DTI changes using individually traced regions of interest outlining the target white matter structure. Our study also extends the characterization of white matter changes to the hypertensive rat MCAO stroke model.

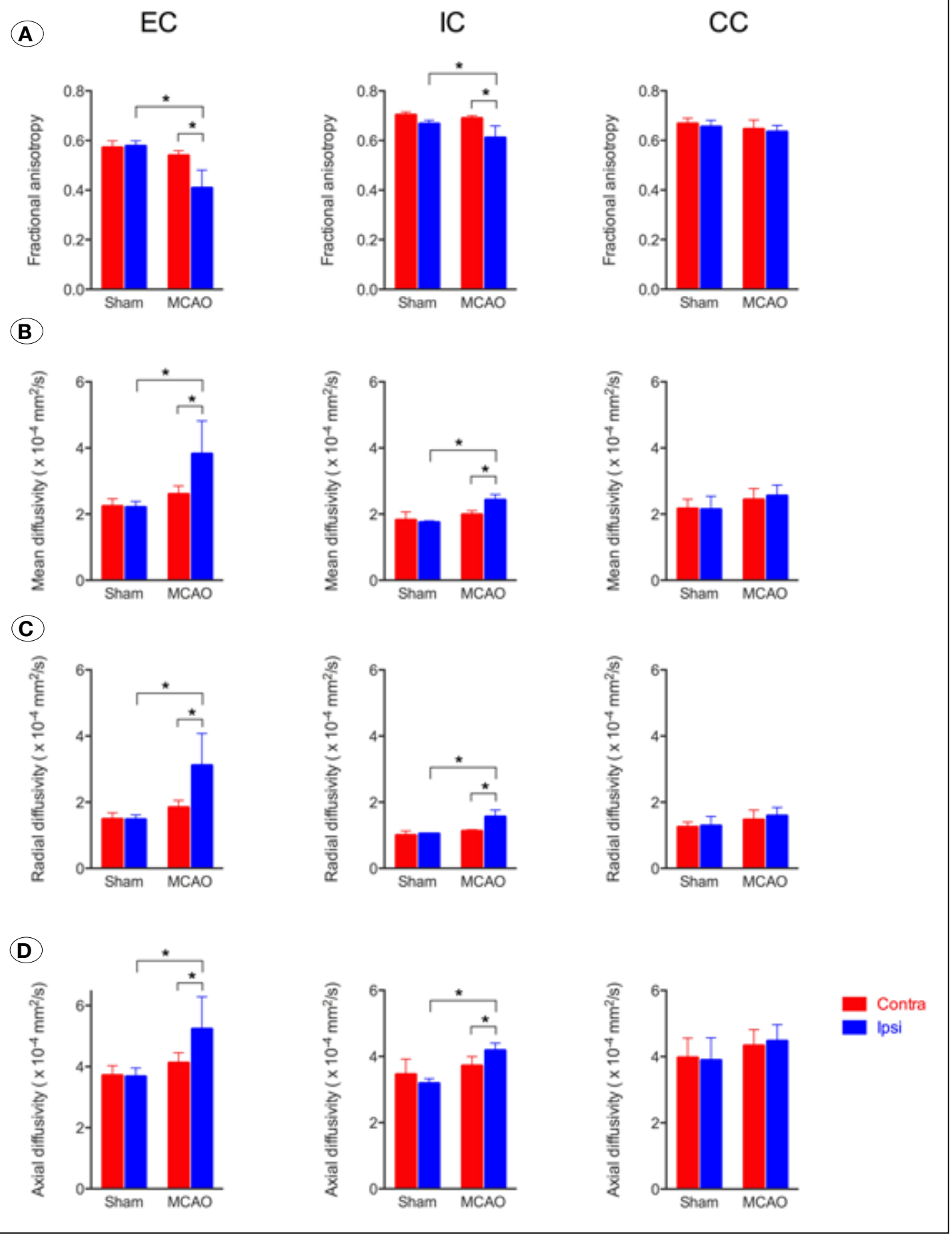

Figure 4:

Measurements of mean fractional anisotropy (FA) (A), mean diffusivity (MD) (B), mean radial diffusivity (RD) (C), and mean axial diffusivity (AD) (D) in ipsilateral (Ipsi) or contralateral (Contra), CC, EC and IC regions of interest, assessed 35 days after MCAO or sham surgery. Error bars represent standard deviations. ${ }^{*} p<0.05$. 

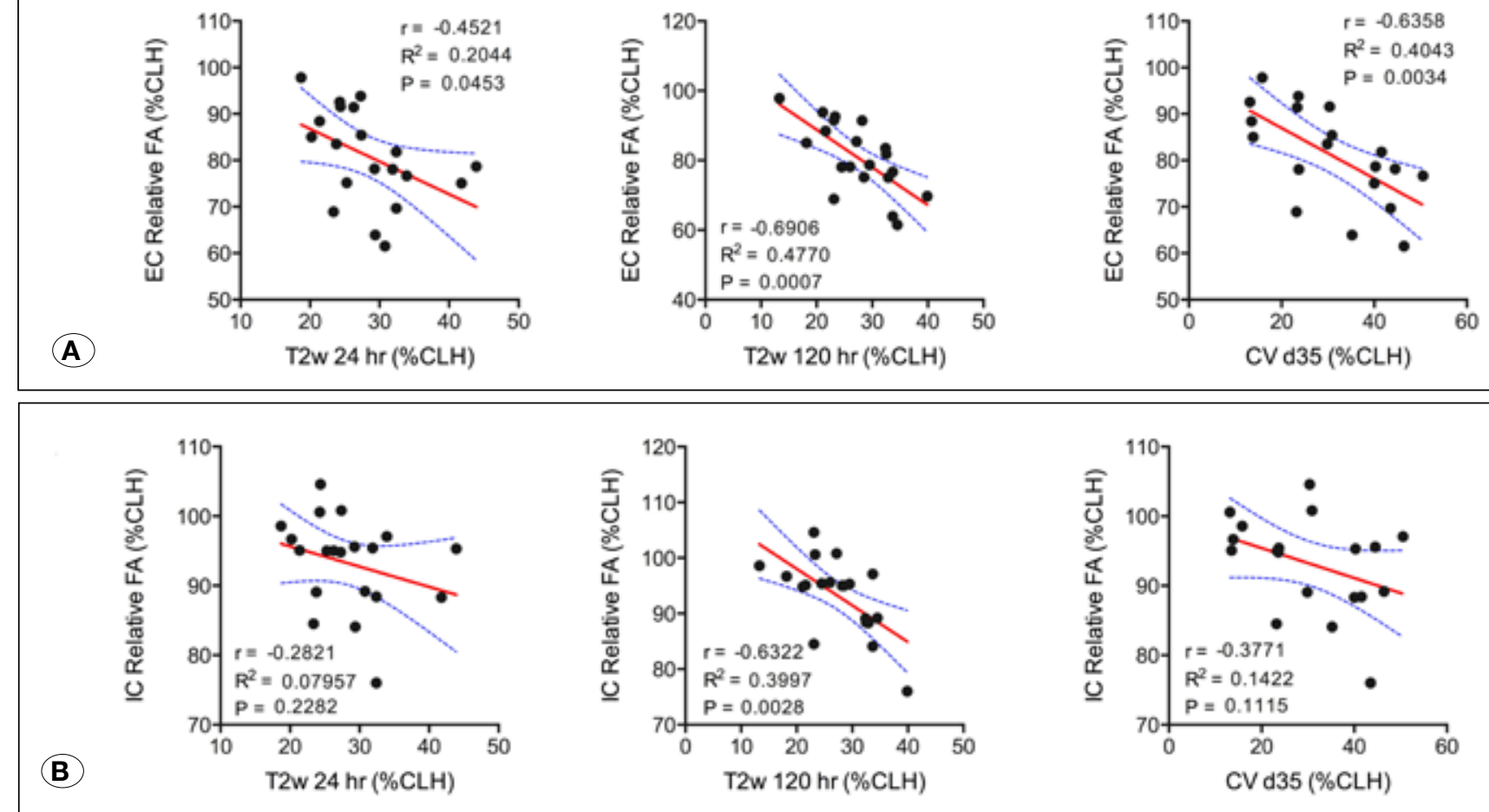

Figure 5: Correlation among T2w-MRI-derived (24 h or $120 \mathrm{~h}$ ) or cresyl violet staining-derived (d35) infarct volumes and relative fractional anisotropy measured 35 days after surgery for (A) EC or (B) IC. Relative FA represents the ratio of mean ipsilateral FA to mean contralateral FA. \%CLH, percent contralateral hemisphere. Pearson's correlation coefficients were calculated for the indicated pairs of measurements. The best fit linear regressions (red) and their $95 \%$ confidence intervals (blue dotted lines) are shown.

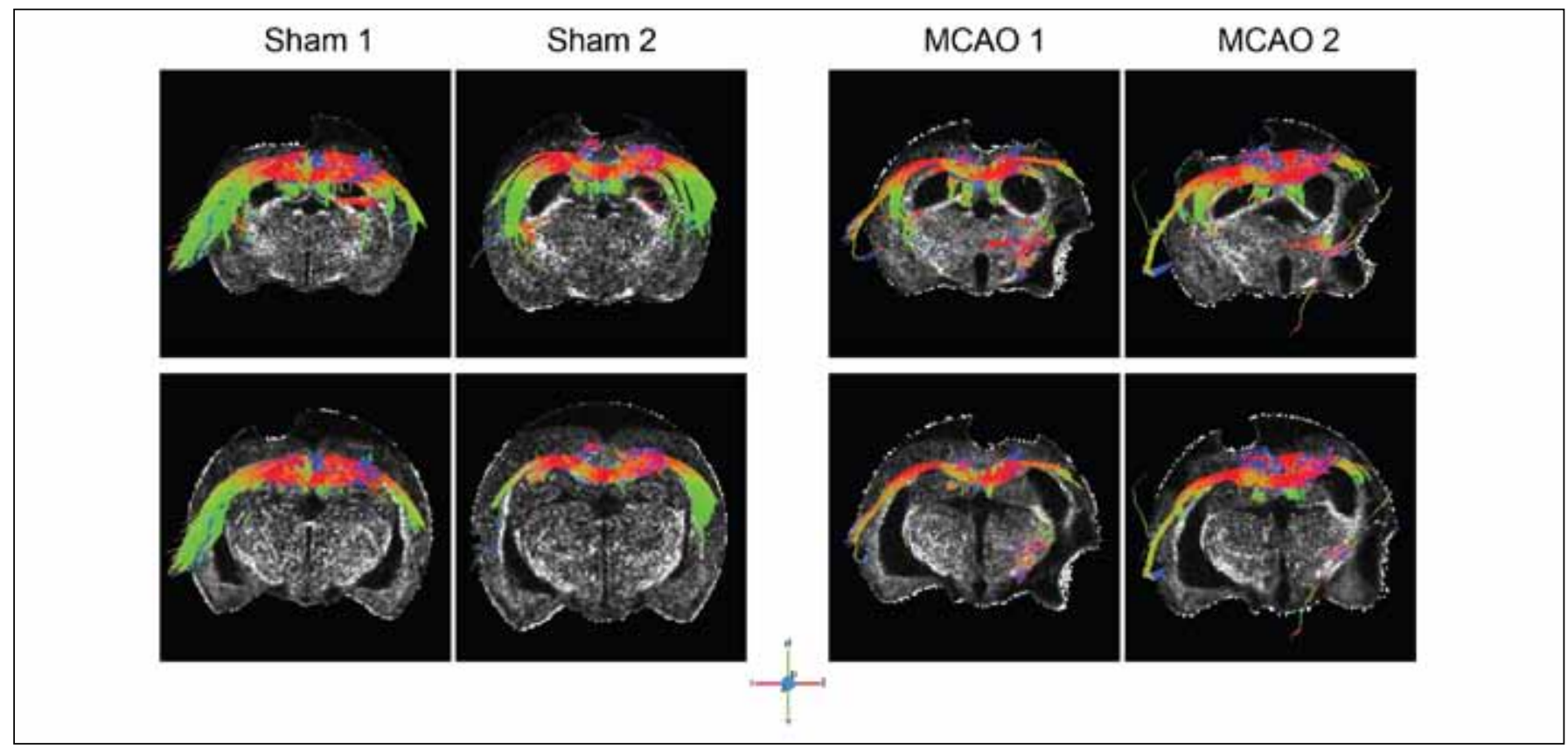

Figure 6: Fiber tractography of sham and MCAO rat brains. Fiber tracking was seeded with the CC regions of interest used in the measurement of diffusivity parameters. Results for two individual rats for each condition are shown against an axial FA image for orientation. The tract directions are color-coded: green, dorsal-ventral; red, left-right; blue, anterior-posterior (rostral-caudal). Note that fiber tracts only appear to extend past the brain border in some locations because the reference FA image slice is located posterior to the fiber projection in this figure. 


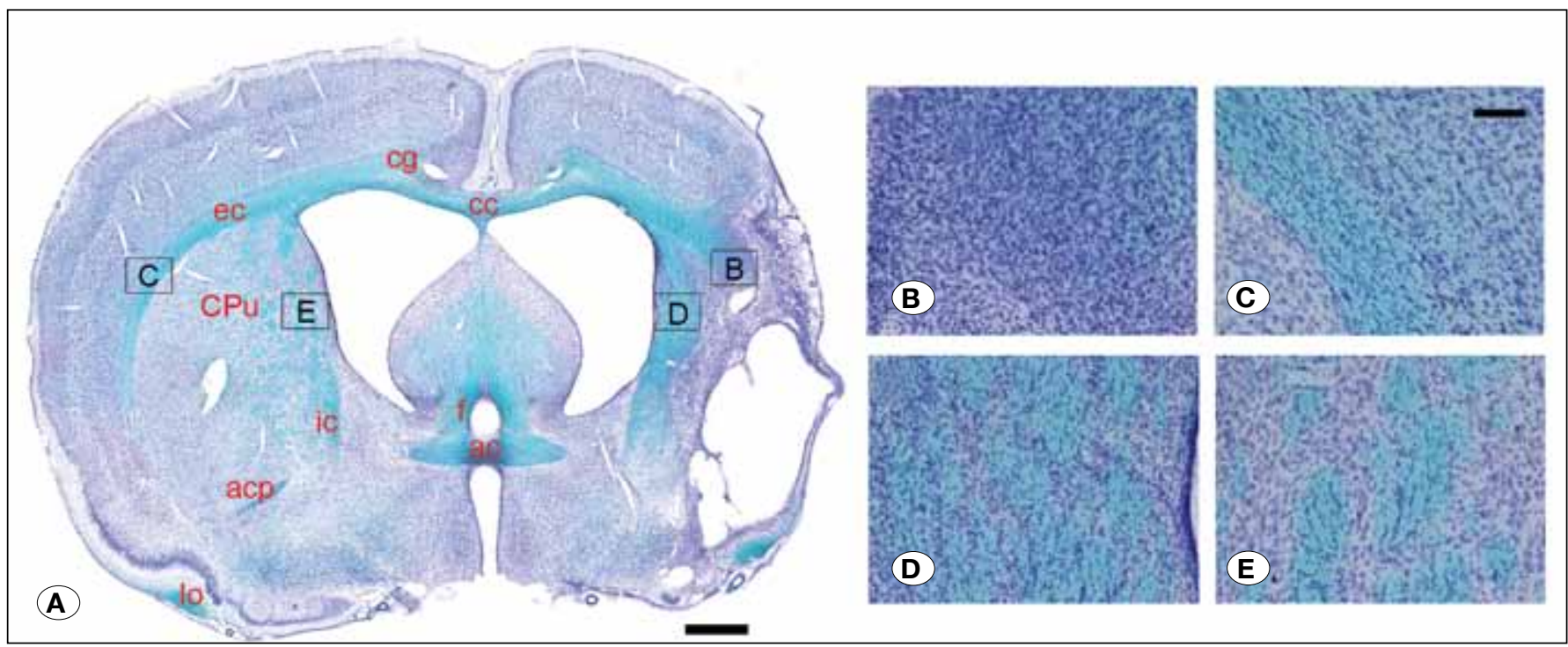

Figure 7: Histological assessment of white matter integrity. (A) Overview image of a rat brain subjected to 60 min MCAO stained with Luxol fast blue and cresyl violet; the entire axial section is stitched from multiple 4X magnification images. The boxed regions indicate fields in panels B-E. The scale bar represents $1 \mathrm{~mm}$. (B) 20X field from the ipsilateral EC. (C) 20X field from the contralateral EC. (D) $20 X$ field from the perilesional caudate/putamen. (E) 20X field from the contralateral caudate/putamen. The scale bar in panel $C$ represents $100 \mu \mathrm{m}$ and applies to panels B-E. Structural abbreviations: ac, anterior commissure; acp, posterior region of the anterior commissure; cc, corpus callosum; cg, cingulum; CPu, caudate/putamen; ec, external capsule; f, fornix; ic, internal capsule; lo, lateral olfactory tract.

Our results clearly demonstrate the loss of white matter integrity in external capsule and IC regions ipsilateral to the site of ischemic injury; this is reflected by the reduced fractional anisotropy in these regions assessed 5 weeks after transient MCAO in hypertensive rats. Not surprisingly, we observed a positive correlation between the extent of ischemic injury as measured histologically or by T2-weighted MRI and the reduction in FA in IC or EC. There was also good agreement between the assessment of external capsule structural integrity visualized by histological staining and the reduction in FA observed by DTI.

Our results are consistent with those that have been observed in other rat strains at various time points following permanent or transient MCAO. For example, Kim et al. (25) initially saw elevated FA during the hyperacute period following either permanent or 30 min MCAO in normotensive Sprague Dawley rats, but FA values subsequently normalized or further decreased by $9 \mathrm{~h}$ or $24 \mathrm{~h}$ post-occlusion, respectively. Van Meer et al. (62) observed decreased FA in ipsilateral IC, EC and cerebral peduncle regions of interest by 3 days after 90 min MCAO in Sprague Dawley rats with both medium and large strokes relative to control rats; corpus callosum also showed reduced $F A$ in this study, but only in rats with large strokes. Interestingly, normalization of FA values similar to those of the control group occurred by 49 days in the medium stroke group but remained below control values at 49 days and 70 days post-stroke in the large stroke group. A developmental increase in FA through early adulthood is to be expected in healthy rats (5).

Using a related technique (diffusion spectrum imaging), Po et al. (44) detected a reduction in generalized FA (gFA, an extension of FA) in the ipsilateral IC versus the healthy contralateral IC at 4 weeks after MCAO in normotensive Wistar rats. They also observed a loss of fibers in the ipsilateral EC at both 1 week and 4 weeks after stroke. This group noted a region of increased gFA between the ischemic lesion and the ipsilateral lateral ventricle, and this region contained altered fiber connections visualized by tractography, suggesting white matter reorganization. This is consistent with our study, in which we too observed alterations in FA and fiber track orientation along the perilesional area, along with increased luxol fast blue staining.

In addition to decreased FA, we also observed alterations in several other diffusion parameters, such as increases in mean, radial and axial diffusivity following MCAO. These changes too are mostly consistent with reports in other models. Mean diffusivity is commonly elevated during the late sub-acute to chronic stages following stroke $(7,15,32,53)$. Increased radial diffusion is suggestive of demyelination injury (52), as the breakdown of the myelin sheath allows increased diffusion of water perpendicular to the long axis of axonal fibers. By contrast, neurodegeneration associated with ischemic injury results in a decrease in axial diffusivity in some studies (51). It has been suggested that $A D$ reflects a time-dependent course of axonal degradation, with the initial decrease in $A D$ reflecting fragmentation of axons, which impedes the longitudinal diffusion of water. Late after injury, axonal fragments are cleared allowing longitudinal diffusion and a normalization or increase in $A D$ values $(11,39)$. Therefore, the increased $A D$ we observed at 5 weeks post-stroke suggests that axonal degeneration contributes to the white matter injury. 
Diffusivity changes may also exhibit complexity among different brain regions. Although the occurrence of hippocampal damage in MCAO models is variable $(23,42,65,67,69)$, decreased hippocampal (CA2/3) MD measured by ex vivo DTI corresponded with cognitive decline at 1 week through 2 months after 90 min MCAO in Sprague Dawley rats (10); this was associated with increased activation of astrocytes and microglia in this hippocampal region. No change in hippocampal FA was observed by these authors, however.

This study has several limitations. One is a relatively small number of animals used in each study group, which could reduce the generalizability of our results. Given the good agreement we've observed with other human and animal studies in the literature, however, we don't believe this to be a significant factor affecting our conclusions. We also were limited by technical constraints from directly comparing in vivo DTI with ex vivo DTI data in a quantitative manner. Additional DTI studies would be useful for examining the long term functional consequences of this experimental stroke model by linking results of behavioral testing to injury or reorganization within specific white matter structures. Similarly, assessing DTI changes associated with vasoactive or neuroprotective pharmaceutical or stem cell treatments after MCAO would be highly interesting.

\section{CONCLUSION}

We believe that this is the first report of ex vivo DTI of hypertensive rats following MCAO. The ability of DTI to monitor microstructural changes in white matter organization may serve as a valuable tool to monitor the impact of therapeutic interventions in this and other animal models of chronic ischemic injury. Furthermore, combining monitoring of white matter reorganization with the evaluation of long term functional and behavioral outcomes may provide insight into the neurological pathways of stroke recovery in model organisms.

\section{ACKNOWLEDGEMENTS}

We thank Beth Rauch of the University of Wisconsin Small Animal Imaging Center and Cheng Guan Koay (University of Wisconsin Department of Medical Physics) for their assistance with MR imaging and data processing. We would also like to thank Arman Quraishi and Tae Kyu Chung for technical assistance. This work was supported by funding from the University of Wisconsin Department of Neurosurgery and NIH grant UL1TR000427 (PF).

\section{REFERENCES}

1. Abdi H: The Bonferroni and Šidák Corrections for Multiple Comparisons. In: Salkind N. (ed). Encyclopedia of Measurement and Statistics. Thousand Oaks, CA: SAGE Publications, 2007:103-107

2. Basser PJ, Mattiello J, LeBihan D: MR diffusion tensor spectroscopy and imaging. Biophys J 66:259-267, 1994
3. Basser PJ, Pajevic S, Pierpaoli C, Duda J, Aldroubi A: In vivo fiber tractography using DT-MRI data. Magn Reson Med 44:625-632, 2000

4. Basser PJ, Pierpaoli C: Microstructural and physiological features of tissues elucidated by quantitative-diffusion-tensor MRI. J Magn Reson B 111:209-219, 1996

5. Bockhorst KH, Narayana PA, Liu R, Ahobila-Vijjula P, Ramu J, Kamel M, Wosik J, Bockhorst T, Hahn K, Hasan KM, PerezPolo JR: Early postnatal development of rat brain: In vivo diffusion tensor imaging. J Neurosci Res 86:1520-1528, 2008

6. Brittain JF, McCabe C, Khatun H, Kaushal N, Bridges LR, Holmes WM, Barrick TR, Graham D, Dominiczak AF, Mhairi Macrae I, Hainsworth AH: An MRI-histological study of white matter in stroke-free SHRSP. J Cereb Blood Flow Metab 33:760-763, 2013

7. Buffon F, Molko N, Hervé D, Porcher R, Denghien I, Pappata $\mathrm{S}$, Le Bihan D, Bousser MG, Chabriat $\mathrm{H}$ : Longitudinal diffusion changes in cerebral hemispheres after MCA infarcts. J Cereb Blood Flow Metab 25:641-650, 2005

8. Cengiz P, Uluc K, Kendigelen P, Akture E, Hutchinson E, Song C, Zhang L, Lee J, Budoff GE, Meyerand E, Sun D, Ferrazzano $P$ : Chronic neurological deficits in mice after perinatal hypoxia and ischemia correlate with hemispheric tissue loss and white matter injury detected by MRI. Dev Neurosci 33:270-279, 2011

9. Chan KC, Khong PL, Lau HF, Cheung PT, Wu EX: Late measures of microstructural alterations in severe neonatal hypoxic-ischemic encephalopathy by MR diffusion tensor imaging. Int J Dev Neurosci 27:607-615, 2009

10. Chin Y, Kishi M, Sekino M, Nakajo F, Abe Y, Terazono Y, Hiroyuki O, Kato F, Koizumi S, Gachet C, Hisatsune T: Involvement of glial $\mathrm{P}_{2} \mathrm{Y}_{1}$ receptors in cognitive deficit after focal cerebral stroke in a rodent model. J Neuroinflammation 10:95, 2013

11. Concha L, Gross DW, Wheatley BM, Beaulieu C: Diffusion tensor imaging of time-dependent axonal and myelin degradation after corpus callosotomy in epilepsy patients. Neuroimage 32:1090-1099, 2006

12. Coutts SB, Simon JE, Tomanek AI, Barber PA, Chan J, Hudon ME, Mitchell JR, Frayne R, Eliasziw M, Buchan AM, Demchuk AM: Reliability of assessing percentage of diffusion-perfusion mismatch. Stroke 34:1681-1683, 2003

13. Coyle P: Different susceptibilities to cerebral infarction in spontaneously hypertensive (SHR) and normotensive Sprague-Dawley rats. Stroke 17:520-525, 1986

14. D'Arceuil HE, Westmoreland S, de Crespigny AJ: An approach to high resolution diffusion tensor imaging in fixed primate brain. Neuroimage 35:553-565, 2007

15. Dijkhuizen RM, van der Marel K, Otte WM, Hoff El, van der Zijden JP, van der Toorn A, van Meer MPA: Functional MRI and diffusion tensor imaging of brain reorganization after experimental stroke. Transl Stroke Res 3:36-43, 2012

16. Dogan A, Baskaya MK, Rao VL, Rao AM, Dempsey RJ: Intraluminal suture occlusion of the middle cerebral artery in Spontaneously Hypertensive rats. Neurol Res 20:265-270, 1998

17. Duverger D, MacKenzie ET: The Quantification of cerebral infarction following focal ischemia in the rat: Influence of strain, arterial pressure, blood glucose concentration, and age. J Cereb Blood Flow Metab 8:449-461, 1988 
18. Engvig A, Fjell AM, Westlye LT, Moberget T, Sundseth $\varnothing$, Larsen VA, Walhovd KB: Memory training impacts short-term changes in aging white matter: A longitudinal diffusion tensor imaging study. Hum Brain Mapp 33:2390-2406, 2012

19. Gorgui J, Gorshkov M, Khan N, Daskalopoulou SS: Hypertension as a risk factor for ischemic stroke in women. Can J Cardiol 30:774-782, 2014

20. Guilfoyle DN, Helpern JA, Lim KO: Diffusion tensor imaging in fixed brain tissue at 7.0 T. NMR Biomed 16:77-81, 2003

21. Jang SH, Cho SH, Kim YH, Han BS, Byun WM, Son SM, Kim $\mathrm{SH}$, Lee SJ: Diffusion anisotropy in the early stages of stroke can predict motor outcome. Restor Neurol Neurosci 23:1117, 2005

22. Jiang Q, Thiffault C, Kramer BC, Ding GL, Zhang L, NejadDavarani SP, Li L, Arbab AS, Lu M, Navia B, Victor SJ, Hong K, Li QJ, Wang SY, Li Y, Chopp M: MRI detects brain reorganization after human umbilical tissue-derived cells (hUTC) treatment of stroke in rat. PLoS ONE 7:e42845, 2012

23. Jin K, Minami M, Lan JQ, Mao XO, Batteur S, Simon RP, Greenberg DA: Neurogenesis in dentate subgranular zone and rostral subventricular zone after focal cerebral ischemia in the rat. Proc Natl Acad Sci USA 98:4710-4715, 2001

24. Jones DK, Horsfield MA, Simmons A: Optimal strategies for measuring diffusion in anisotropic systems by magnetic resonance imaging. Magn Reson Med 42:515-525, 1999

25. Kim JH, Na DG, Chang KH, Song IC, Choi SH, Son KR, Kim $\mathrm{KW}$, Sohn $\mathrm{CH}$ : Serial MR analysis of early permanent and transient ischemia in rats: Diffusion tensor imaging and high b value diffusion weighted imaging. Korean J Radiol 14:307315, 2013

26. Kim JH, Trinkaus K, Ozcan A, Budde MD, Song SK: Postmortem delay does not change regional diffusion anisotropy characteristics in mouse spinal cord white matter. NMR Biomed 20:352-359, 2007

27. Klawiter EC, Schmidt RE, Trinkaus K, Liang HF, Budde MD, Naismith RT, Song SK, Cross AH, Benzinger TL: Radial diffusivity predicts demyelination in ex vivo multiple sclerosis spinal cords. Neuroimage 55:1454-1460, 2011

28. Koay CG, Chang L-C, Carew JD, Pierpaoli C, Basser PJ: A unifying theoretical and algorithmic framework for least squares methods of estimation in diffusion tensor imaging. $J$ Magn Reson 182:115-125, 2006

29. Koizumi J-I, Yoshida Y, Nakazawa T, Ooneda G: Experimental studies of ischemic brain edema. 1. A new experimental model of cerebral embolism in rats in which recirculation can be introduced in the ischemic area. Japanese Journal of Stroke 8:1-8, 1986

30. Lehmann HC, Zhang J, Mori S, Sheikh KA: Diffusion tensor imaging to assess axonal regeneration in peripheral nerves. Exp Neurol 223:238-244, 2010

31. Lin TN, He YY, Wu G, Khan M, Hsu CY: Effect of brain edema on infarct volume in a focal cerebral ischemia model in rats. Stroke 24:117-121, 1993

32. Liu Y, D'Arceuil HE, Westmoreland S, He J, Duggan M, Gonzalez RG, Pryor J, de Crespigny AJ: Serial diffusion tensor MRI after transient and permanent cerebral ischemia in nonhuman primates. Stroke 38:138-145, 2007
33. Liu YP, Seçkin H, Izci Y, Du ZW, Yan YP, Baskaya MK: Neuroprotective effects of mesenchymal stem cells derived from human embryonic stem cells in transient focal cerebral ischemia in rats. J Cereb Blood Flow Metab 29:780-791, 2009

34. Longa EZ, Weinstein PR, Carlson S, Cummins R: Reversible middle cerebral artery occlusion without craniectomy in rats. Stroke 20:84-91, 1989

35. López-Gil X, Amat-Roldan I, Tudela R, Castañé A, PratsGalino A, Planas AM, Farr TD, Soria G: DWI and complex brain network analysis predicts vascular cognitive impairment in spontaneous hypertensive rats undergoing executive function tests. Front Aging Neurosci 6:167, 2014

36. Mori S, Zhang J: Principles of diffusion tensor imaging and its applications to basic neuroscience research. Neuron 51:527539, 2006

37. Moseley ME, Kucharczyk J, Asgari HS, Norman D: Anisotropy in diffusion-weighted MRI. Magn Reson Med 19:321-326, 1991

38. Møller M, Frandsen J, Andersen G, Gjedde A, VestergaardPoulsen P, Ostergaard L: Dynamic changes in corticospinal tracts after stroke detected by fibretracking. J Neurol Neurosurg Psychiatr 78:587-592, 2007

39. Nave Della R, Ginestroni A, Diciotti S, Salvatore E, Soricelli A, Mascalchi M: Axial diffusivity is increased in the degenerating superior cerebellar peduncles of Friedreich's ataxia. Neuroradiology 53:367-372, 2011

40. O'Donnell MJ, Xavier D, Liu L, Zhang H, Chin SL, RaoMelacini $P$, Rangarajan S, Islam S, Pais P, McQueen MJ, Mondo C, Damasceno A, Lopez-Jaramillo P, Hankey GJ, Dans AL, Yusoff K, Truelsen T, Diener HC, Sacco RL, Ryglewicz D, Czlonkowska A, Weimar C, Wang X, Yusuf S, Investigators I: Risk factors for ischaemic and intracerebral haemorrhagic stroke in 22 countries (the INTERSTROKE study): A casecontrol study. Lancet 376:112-123, 2010

41. Oguz I, McMurray MS, Styner M, Johns JM: The translational role of diffusion tensor image analysis in animal models of developmental pathologies. Dev Neurosci 34:5-19, 2012

42. Ozdemir YG, Bolay H, Erdem E, Dalkara T: Occlusion of the MCA by an intraluminal filament may cause disturbances in the hippocampal blood flow due to anomalies of circle of Willis and filament thickness. Brain Res 822:260-264, 1999

43. Pitkonen M, Abo-Ramadan U, Marinkovic I, Pedrono E, Hasan KM, Strbian D, Durukan A, Tatlisumak T: Long-term evolution of diffusion tensor indices after temporary experimental ischemic stroke in rats. Brain Res 1445:103-110, 2012

44. Po C, Kalthoff D, Kim YB, Nelles M, Hoehn M: White matter reorganization and functional response after focal cerebral ischemia in the rat. PLoS ONE 7:e45629, 2012

45. Puig J, Blasco G, Daunis-I-Estadella J, Thomalla G, Castellanos M, Figueras $\mathrm{J}$, Remollo $\mathrm{S}$, van Eendenburg C, Sánchez-González J, Serena J, Pedraza S: Decreased corticospinal tract fractional anisotropy predicts long-term motor outcome after stroke. Stroke 44(7): 2016-21018, 2013

46. Puig J, Pedraza S, Blasco G, Daunis-I-Estadella J, Prados F, Remollo S, Prats-Galino A, Soria G, Boada I, Castellanos $M$, Serena J: Acute damage to the posterior limb of the internal capsule on diffusion tensor tractography as an early imaging predictor of motor outcome after stroke. AJNR Am J Neuroradiol 32:857-863, 2011 
47. Rane S, Duong TQ: Comparison of in vivo and ex vivo diffusion tensor imaging in rhesus macaques at short and long diffusion times. Open Neuroimag J 5:172-178, 2011

48. Ruest T, Holmes WM, Barrie JA, Griffiths IR, Anderson TJ, Dewar D, Edgar JM: High-resolution diffusion tensor imaging of fixed brain in a mouse model of Pelizaeus-Merzbacher disease: Comparison with quantitative measures of white matter pathology. NMR Biomed 24:1369-1379, 2011

49. Scholz J, Klein MC, Behrens TEJ, Johansen-Berg H: Training induces changes in white-matter architecture. Nat Neurosci 12:1370-1371, 2009

50. Sobesky J: Refining the mismatch concept in acute stroke: Lessons learned from PET and MRI. J Cereb Blood Flow Metab 32:1416-1425, 2012

51. Song SK, Sun SW, Ju WK, Lin SJ, Cross AH, Neufeld AH: Diffusion tensor imaging detects and differentiates axon and myelin degeneration in mouse optic nerve after retinal ischemia. Neuroimage 20:1714-1722, 2003

52. Song SK, Sun SW, Ramsbottom MJ, Chang C, Russell J, Cross AH: Dysmyelination revealed through MRI as increased radial (but unchanged axial) diffusion of water. Neuroimage 17:1429-1436, 2002

53. Sotak $\mathrm{CH}$ : The role of diffusion tensor imaging in the evaluation of ischemic brain injury - a review. NMR Biomed 15:561-569, 2002

54. Sun SW, Liang HF, Le TQ, Armstrong RC, Cross AH, Song SK: Differential sensitivity of in vivo and ex vivo diffusion tensor imaging to evolving optic nerve injury in mice with retinal ischemia. Neuroimage 32:1195-1204, 2006

55. Sun SW, Liang HF, Xie M, Oyoyo U, Lee A: Fixation, not death, reduces sensitivity of DTI in detecting optic nerve damage. Neuroimage 44:611-619, 2009

56. Sun SW, Neil JJ, Liang HF, He YY, Schmidt RE, Hsu CY, Song SK: Formalin fixation alters water diffusion coefficient magnitude but not anisotropy in infarcted brain. Magn Reson Med 53:1447-1451, 2005

57. Sun SW, Neil JJ, Song SK: Relative indices of water diffusion anisotropy are equivalent in live and formalin-fixed mouse brains. Magn Reson Med 50:743-748, 2003

58. Swanson RA, Morton MT, Tsao-Wu G, Savalos RA, Davidson C, Sharp FR: A semiautomated method for measuring brain infarct volume. J Cereb Blood Flow Metab 10:290-293, 1990

59. Thomalla G, Glauche V, Koch MA, Beaulieu C, Weiller C, Röther J: Diffusion tensor imaging detects early Wallerian degeneration of the pyramidal tract after ischemic stroke. Neuroimage 22:1767-1774, 2004

60. Uluc K, Miranpuri A, Kujoth GC, Akture E, Baskaya MK: Focal cerebral ischemia model by endovascular suture occlusion of the middle cerebral artery in the rat. J Vis Exp (48)pii:1978, 2011
61. van der Zijden JP, van der Toorn A, van der Marel K, Dijkhuizen $\mathrm{RM}$ : Longitudinal in vivo MRI of alterations in perilesional tissue after transient ischemic stroke in rats. Exp Neurol 212:207-212, 2008

62. van Meer MPA, Otte WM, van der Marel $\mathrm{K}$, Nijboer $\mathrm{CH}$, Kavelaars A, van der Sprenkel JWB, Viergever MA, Dijkhuizen RM: Extent of bilateral neuronal network reorganization and functional recovery in relation to stroke severity. J Neurosci 32:4495-4507, 2012

63. Virley D, Beech JS, Smart SC, Williams SC, Hodges $H$, Hunter AJ: A temporal MRI assessment of neuropathology after transient middle cerebral artery occlusion in the rat: Correlations with behavior. J Cereb Blood Flow Metab 20:563-582, 2000

64. Werring DJ, Toosy AT, Clark CA, Parker GJ, Barker GJ, Miller $\mathrm{DH}$, Thompson AJ: Diffusion tensor imaging can detect and quantify corticospinal tract degeneration after stroke. J Neurol Neurosurg Psychiatr 69:269-272, 2000

65. Xie M, Yi C, Luo X, Xu S, Yu Z, Tang Y, Zhu W, Du Y, Jia L, Zhang Q, Dong Q, Zhu W, Zhang X, Bu B, Wang W: Glial gap junctional communication involvement in hippocampal damage after middle cerebral artery occlusion. Ann Neurol 70:121-132, 2011

66. Yang Q, Tress BM, Barber PA, Desmond PM, Darby DG, Gerraty RP, Li T, Davis SM: Serial study of apparent diffusion coefficient and anisotropy in patients with acute stroke. Stroke 30:2382-2390, 1999

67. Yang SH, Shetty RA, Liu R, Sumien N, Heinrich KR, Rutledge $M$, Thangthaeng $N$, Brun-Zinkernagel AM, Forster $M J$ : Endovascular middle cerebral artery occlusion in rats as a model for studying vascular dementia. AGE 28:297-307, 2006

68. Yeh FC, Verstynen TD, Wang Y, Fernández-Miranda JC, Tseng WYI: Deterministic diffusion fiber tracking improved by quantitative anisotropy. PLoS ONE 8:e80713, 2013

69. Yonemori F, Yamaguchi T, Yamada H, Tamura A: Spatial cognitive performance after chronic focal cerebral ischemia in rats. J Cereb Blood Flow Metab 19:483-494, 1999

70. Zhang J, Jones MV, Mc Mahon MT, Mori S, Calabresi PA: In vivo and ex vivo diffusion tensor imaging of cuprizoneinduced demyelination in the mouse corpus callosum. Magn Reson Med 67:750-759, 2012

71. Zheng X, Schlaug G: Structural white matter changes in descending motor tracts correlate with improvements in motor impairment after undergoing a treatment course of tDCS and physical therapy. Front Hum Neurosci 9:229, 2015 